\title{
Meshless Polyharmonic Div-Curl Reconstruction
}

\author{
M. N. Benbourhim ${ }^{1}$ and A. Bouhamidi ${ }^{2 *}$ \\ ${ }^{1}$ Institute of Mathematics of Toulouse, University of Paul Sabatier, \\ 118, route de Narbonne, F-31062 Toulouse Cedex 9, France \\ ${ }^{2}$ University of Lille Nord de France, ULCO, L.M.P.A, \\ 50, rue F. Buisson BP 699, F-62228 Calais Cedex, France
}

\begin{abstract}
In this paper, we will discuss the meshless polyharmonic reconstruction of vector fields from scattered data, possibly, contaminated by noise. We give an explicit solution of the problem. After some theoretical framework, we discuss some numerical aspect arising in the problems related to the reconstruction of vector fields.
\end{abstract}

Key words: approximation theory, meshless approximation methods, radial basis functions AMS subject classification: 41A15, 41A29, 41A65, 65D05

\section{Introduction}

Vector field reconstruction is a problem arising in many scientific applications. These include, for example, fluid mechanic, electromagnetic, meteorology and optic flow analysis. When considering the approximation of vector fields, a key problem is how to correlate its components. It has been observed, particularly for meteorological problems, that if no inter-component correlation is assumed the approximating field may give unrealistic results. In this paper, we present a brief discussion on the reconstruction of vector fields from scattered data points by using polyharmonic meshless approximation. The theory of plyharmonic div-curl reconstruction was developed in a general framework based upon earlier results on generalized pseudo-polyharmonic approximation [1].

*Corresponding author. E-mail: a.bouhamidi@1mpa.univ-littoral.fr 


\section{The functional space}

Let $n, m \in \mathbb{N}^{\star}:=\mathbb{N} \backslash\{0\}$ with $n \geq 2$, and consider the space

$$
\begin{aligned}
X^{m}\left(\mathbb{R}^{n} ; \mathbb{R}^{n}\right)=\left\{u=\left(u_{1}, \ldots, u_{n}\right)^{T}\right. & \in \mathscr{D}^{\prime}\left(\mathbb{R}^{n} ; \mathbb{R}^{n}\right): \\
\forall \alpha & \left.\in \mathbb{Z}_{+}^{n},|\alpha|=m, \quad \partial^{\alpha} u_{i} \in L^{2}\left(\mathbb{R}^{n}\right), i=1, \ldots, n\right\},
\end{aligned}
$$

where $\mathscr{D}^{\prime}\left(\mathbb{R}^{n} ; \mathbb{R}^{n}\right)$ is the space of the vector-valued distributions on $\mathbb{R}^{n}$, and the notation $u^{T}$ stands for the transpose of $u$. We assume that

$$
m>\frac{n}{2}
$$

The space $X^{m}\left(\mathbb{R}^{n} ; \mathbb{R}^{n}\right)$ is equipped with the following semi-scalar product and its associated seminorm

$$
(u \mid v)_{m}=\sum_{|\alpha|=m} \frac{m !}{\alpha !} \int_{\mathbb{R}^{n}} \partial^{\alpha} u^{T}(\xi) \partial^{\alpha} v(\xi) d \xi, \quad|u|_{m}=\sqrt{(u \mid u)_{m}} .
$$

The null space associated to the semi-scalar product is the space, denoted by $\Pi_{m-1}\left(\mathbb{R}^{n} ; \mathbb{R}^{n}\right)$ of vector-valued polynomials of $n$-variables with degree $\leq m-1$.

Let $N$ be a nonnegative integer and suppose we are given a collection of $N$ distinct points $x_{1}, \ldots, x_{N}$ in $\mathbb{R}^{n}$, such that the set $\Omega_{N}=\left\{x_{1}, \ldots, x_{N}\right\}$ contains a $\Pi_{m-1}\left(\mathbb{R}^{n}\right)$-unisolvent subset. We recall that a set $\Omega$ is $\Pi_{m-1}\left(\mathbb{R}^{n}\right)$-unisolvent if any polynomial in $\Pi_{m-1}\left(\mathbb{R}^{n}\right)$ which vanishes on $\Omega$ is identically zero. We consider the operator $L: X^{m, s}\left(\mathbb{R}^{n} ; \mathbb{R}^{n}\right) \longrightarrow \mathbb{R}^{N \times n}$ given for $u=$ $\left(u_{1}, \ldots, u_{n}\right)^{T}$ by

$$
L u=\left(\begin{array}{ccc}
u_{1}\left(x_{1}\right) & \ldots & u_{n}\left(x_{1}\right) \\
\vdots & \ddots & \vdots \\
u_{1}\left(x_{N}\right) & \ldots & u_{n}\left(x_{N}\right)
\end{array}\right)
$$

The space $X^{m}\left(\mathbb{R}^{n} ; \mathbb{R}^{n}\right)$ is now equipped with the scalar product associated to the operator $L$ and defined by

$$
(u \mid v)_{L, m}=(u \mid v)_{L, m}+\langle L u \mid L v\rangle_{N \times n} .
$$

The associated norm is denoted by $\|u\|_{L, m}=\sqrt{(u \mid u)_{L, m}}$. Here the notation $\langle. \mid .\rangle_{N \times n}$ stands for the Frobenius scalar product $\left\langle z \mid z^{\prime}\right\rangle_{N \times n}=\operatorname{trace}\left(z^{T} z^{\prime}\right)$. Its associated norm is denoted by $\|.\|_{N \times n}$ in $\mathbb{R}^{N \times n}$. The following proposition gives some topological properties of the space $X^{m}\left(\mathbb{R}^{n} ; \mathbb{R}^{n}\right)$ equipped with the scalar product (2.5) and its associated norm.

Proposition 1. The space $X^{m}\left(\mathbb{R}^{n} ; \mathbb{R}^{n}\right)$ endowed with the scalar product $(. \mid .)_{L, m}$ is a Hilbert space. The following continuous inclusions hold, for all integer $k$ such that $k<m-n / 2$,

$$
X^{m}\left(\mathbb{R}^{n} ; \mathbb{R}^{n}\right) \hookrightarrow \mathscr{S}^{\prime}\left(\mathbb{R}^{n} ; \mathbb{R}^{n}\right), \quad X^{m}\left(\mathbb{R}^{n} ; \mathbb{R}^{n}\right) \hookrightarrow \mathscr{C}^{k}\left(\mathbb{R}^{n} ; \mathbb{R}^{n}\right) .
$$

Furthermore, the space $\mathscr{D}\left(\mathbb{R}^{n} ; \mathbb{R}^{n}\right)+\Pi_{m-1}\left(\mathbb{R}^{n} ; \mathbb{R}^{n}\right)$ is dense in $X^{m}\left(\mathbb{R}^{n} ; \mathbb{R}^{n}\right)$.

Proof. See [1]. 


\section{Polyharmonic div-curl approximation}

The div and curl operators are defined by

$$
\operatorname{div} u=\nabla^{T} \cdot u=\sum_{i=1}^{n} \partial_{i} u_{i}, \quad \operatorname{curl} u=\nabla \cdot u^{T}-\left(\nabla \cdot u^{T}\right)^{T}=\left(\partial_{i} u_{j}-\partial_{j} u_{i}\right)_{1 \leq i, j \leq n}
$$

where $\nabla=\left(\partial_{1}, \ldots, \partial_{n}\right)^{T}$ stands for the gradient operator and $u=\left(u_{1}, \ldots, u_{n}\right)^{T}$ is a vector-valued distribution. The general definition of the curl is classical in multidimensional harmonic analysis, see $[3,6]$. Let $\rho>0$ denote a positive real parameter. We consider the bilinear forms $D_{m}, R_{m}$ and $M_{m}^{\rho}$ given by

$$
\begin{aligned}
& D_{m}(u, v)=\sum_{|\alpha|=m-1} \frac{(m-1) !}{\alpha !} \int_{\mathbb{R}^{n}} \partial^{\alpha}(\operatorname{div} u)(\xi) \partial^{\alpha}(\operatorname{div} v)(\xi) d \xi \\
& R_{m}(u, v)=\frac{1}{2} \sum_{|\alpha|=m-1} \frac{(m-1) !}{\alpha !} \int_{\mathbb{R}^{n}}\left\langle\partial^{\alpha}(\operatorname{curl} u)(\xi) \mid \partial^{\alpha}(\operatorname{curl} v)(\xi)\right\rangle_{n \times n} d \xi, \\
& M_{m}^{\rho}(u, v)=\rho D_{m}(u, v)+R_{m}(u, v) .
\end{aligned}
$$

The quadratic forms associated to $D_{m}, R_{m}$ and $M_{m}^{\rho}$ are called the div-energy, the curl-energy and the div-curl energy, respectively.

Let $Z \in \mathbb{R}^{N \times n}$ be a $N \times n$ matrix, $\rho>0$, and $\lambda \geq 0$ and consider the following approximation problem:

$$
\min _{v \in I_{\lambda}^{m}(Z)}\left(M_{m}^{\rho}(v, v)+\lambda\|L[v]-Z\|_{N \times n}^{2}\right),
$$

where $I_{0}^{m}(Z)=\left\{v \in X^{m}\left(\mathbb{R}^{n} ; \mathbb{R}^{n}\right): L(v)=Z\right\}$ and $I_{\lambda}^{m}(Z)=X^{m}\left(\mathbb{R}^{n} ; \mathbb{R}^{n}\right)$ for $\lambda>0$.

We have the following

Theorem 2. For all $Z \in \mathbb{R}^{N \times n}, \rho>0$ and $\lambda \geq 0$. The problem (3.1) has a unique solution, denoted by $\sigma_{\lambda}$. The solution $\sigma_{\lambda}$ is the unique element in $X^{m}\left(\mathbb{R}^{n} ; \mathbb{R}^{n}\right)$ characterized by

$$
M_{m}^{\rho}\left(\sigma_{0}, v\right)=0, \forall v \in \operatorname{ker}(L)
$$

for $\lambda=0$, and for $\lambda>0$ :

$$
M_{m}^{\rho}\left(\sigma_{\lambda}, v\right)+\lambda\left\langle L\left[\sigma_{\lambda}\right]-Z \mid L[v]\right\rangle_{N \times n}=0, \forall v \in X^{m}\left(\mathbb{R}^{n} ; \mathbb{R}^{n}\right) .
$$

Proof. The results are obtained from some arguments using the variational spline theory, see [1].

Let us consider the function $K_{m}$, see [2,5], defined by

$$
K_{m}(x)= \begin{cases}c_{1, m}|| x \|^{2 m-n} \log (\|x\|) & \text { if } m-n / 2 \in \mathbb{N}^{\star} \\ c_{2, m} \mid\|x\|^{2 m-n} & \text { if } m-n / 2 \notin \mathbb{N}^{\star}\end{cases}
$$


The values of the constants $c_{1, m}$ and $c_{2, m}$ are such that the function $K_{m}$ satisfies the following relation $\Delta^{m} K_{m}=\delta$, where $\delta$ is the Dirac measure at the origin. For $\rho>0$, we introduce the matrix-function $F_{m}^{\rho}$ defined by

$$
F_{m, s}^{\rho}=\left(-\delta_{l, k} \Delta K_{m+1}+\left(1-\frac{1}{\rho}\right) \partial_{l, k}^{2} K_{m+1}\right)_{1 \leq l, k \leq n},
$$

where the notation $\delta_{l, k}$ stands for the Kronecker symbol and $\partial_{l, k}^{2}$ stands for the second partial derivative.

Let $d=(m+n-1) ! /(n !(m-1) !)$ be the dimension and $\left(q_{1}, \ldots, q_{d}\right)$ be a basis of the scalar space $\Pi_{m-1}\left(\mathbb{R}^{n}\right)$, respectively. The following theorem gives an explicit expression of the unique solution $\sigma_{\lambda}$ of Problem (3.1):

Theorem 3. There is a unique vector-measure $\omega_{\lambda}$ of the form

$$
\omega_{\lambda}=\left(\sum_{i=1}^{N} \alpha_{i, 1}^{(\lambda)} \delta_{x_{i}}, \ldots, \sum_{i=1}^{N} \alpha_{i n}^{(\lambda)} \delta_{x_{i}}\right)^{T}
$$

orthogonal to the space $\Pi_{m-1}\left(\mathbb{R}^{n} ; \mathbb{R}^{n}\right)$, such that $\sigma_{\lambda}-F_{m, s}^{\rho} * \omega_{\lambda} \in \Pi_{m-1}\left(\mathbb{R}^{n} ; \mathbb{R}^{n}\right)$. Thus, there are unique vectors $V_{1, \lambda}, \ldots, V_{N, \lambda} \in \mathbb{R}^{n}$ and $W_{1, \lambda}, \ldots, W_{d, \lambda} \in \mathbb{R}^{n}$ such that the unique solution $\sigma_{\lambda}$ of Problem (3.1) is explicitly given by

$$
\sigma_{\lambda}(x)=\sum_{i=1}^{N} F_{m, s}^{\rho}\left(x-x_{i}\right) V_{i, \lambda}+\sum_{j=1}^{d} q_{j}(x) W_{j, \lambda}, \quad \forall x \in \mathbb{R}^{n},
$$

with the orthogonality conditions $\sum_{i=1}^{N} q_{j}\left(x_{i}\right) V_{i \lambda}=0$, for $j=1, \ldots, d$.

Proof. The result is a consequence of Theorem 2, see [1].

\section{Numerical example}

In Figure 1, we give an original vector field and the pseudo-polyharmonic vector fields approximating the original vector field for $\rho=0.4, \lambda=0$ and for a small value $N=10$ and a large value $N=1000$ of the scattered data points. We point out that when $N$ becomes large the reconstructed vector field is similar to the original vector field. The computed relative error was $R_{e} \simeq 1.5713 \times 10^{-1}$ for $N=10$ and $R_{e} \simeq 1.0403 \times 10^{-4}$ for $N=1000$.

\section{References}

[1] M. N. Benbourhim, A. Bouhamidi. Pseudo-polyharmonic vectorial approximation for divcurl and elastic semi-norms. Numer. Math., 109 (2008), No. 3, 333-364. 

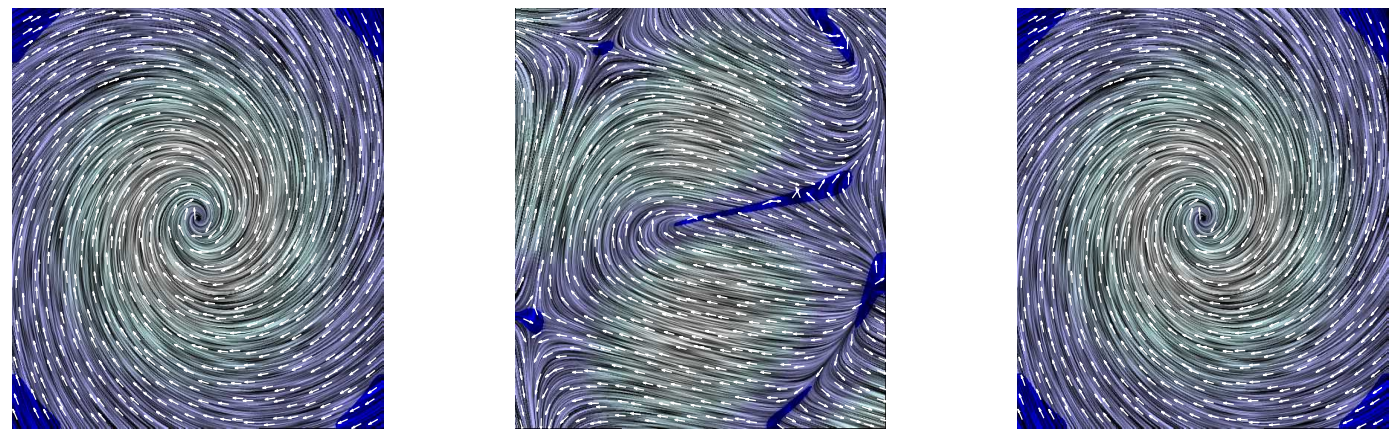

Figure 1: Original field (left), reconstructed fields with $N=10$ (center) and $N=1000$ (right)

[2] J. Duchon. Splines minimizing rotation-invariant seminorms in Sobolev spaces. In constructive theory of functions of several variables, eds. W. Schempp and K. Zeller, Lecture notes in mathematics, vol. 571, Springer-Verlag, Berlin, (1977), 85-100.

[3] T. Iwaniec, C. Sbordone. Quasiharmonic fields. Ann. I. H. Poincaré-AN 18, 5 (2001), 519572.

[4] J. Peetre. Espaces d'interpolation et théorème de Soboleff. Ann. Inst. Fourier, Grenoble, 16 (1966), 279-317.

[5] L. Schwartz. Théorie des distibutions. Hermann, Paris, 1966.

[6] E. Stein. Singular integrals and differentiability properties of functions. Princeton University Press, 1970. 